\title{
BMJ Open Quality Optimisation of lipids for prevention of cardiovascular disease in a primary care
}

\author{
Smita Bakhai, Aishwarya Bhardwaj, Parteet Sandhu, Jessica L. Reynolds
}

To cite: Bakhai S, Bhardwaj $A$ Sandhu P, et al. Optimisation of lipids for prevention of cardiovascular disease in a primary care. BMJ Open Quality 2018;7:e000071. doi:10.1136/ bmjoq-2017-000071

Received 28 March 2017 Revised 11 May 2018 Accepted 23 June 2018
Check for updates

(C) Author(s) (or their employer(s)) 2018. Re-use permitted under CC BY-NC. No commercial re-use. See rights and permissions. Published by BMJ.

Internal Medicine, University at Buffalo, the State University of New York, Buffalo, New York, USA

Correspondence to Dr Smita Bakhai; sybakhai@buffalo.edu

\section{ABSTRACT}

The 2013 American College of Cardiology/American Heart Association (ACC/AHA) guidelines focus on atherosclerotic cardiovascular disease (ASCVD) risk reduction, using a Pooled Cohort Equation to calculate a patient's 10-year risk score, which is used to guide initiation of statin therapy. We identified a gap of evidence-based treatment for hyperlipidaemia in the Internal Medicine Clinic. Therefore, the aim of this study was to increase calculation of ASCVD risk scores in patients between the ages of 40 and 75 years from a baseline rate of less than $1 \%$ to $10 \%$, within 12 months, for primary prevention of ASCVD. Root cause analysis was performed to identify materials/ methods, provider and patient-related barriers. Plan-DoStudy-Act cycles included: (1) creation of customised workflow in electronic health records for documentation of calculated ASCVD risk score; (2) physician education regarding guidelines and electronic health record workflow; (3) refresher training for residents and a chart alert and (4) patient education and physician reminders. The outcome measures were ASCVD risk score completion rate and percentage of new prescriptions for statin therapy. Process measures included lipid profile order and completion rates. Increase in patient wait time, and blood test and medications costs were the balanced measures. We used weekly statistical process control charts for data analysis. The average ASCVD risk completion rate was $14.2 \%$. The mean ASCVD risk completion rate was $4.0 \%$. In eligible patients, the average lipid profile completion rate was $18 \%$. ASCVD risk score completion rate was 33\% 1-year postproject period. A team-based approach led to a sustainable increase in ASCVD risk score completion rate. Lack of automation in ASCVD risk score calculation and physician prompts in electronic health records were identified as major barriers. Furthermore, the team identified multiple barriers to lipid blood tests and treatment of increased ASCVD risk based on ACC/AHA guidelines.

\section{INTRODUCTION}

Problem

Atherosclerotic cardiovascular disease (ASCVD) remains the leading cause of mortality in the world with approximately 17.7 million annual deaths. ${ }^{1}$ The American College of Cardiology/American Heart Association (ACC/AHA) released guidelines on the treatment of blood lipids to reduce ASCVD in adults in 2013. ${ }^{2-6}$ These guidelines include a Pooled Cohort Equation risk calculator that uses age, gender, race, diabetes, hypertension and smoking history, lipid levels and blood systolic pressure to guide primary prevention methods of ASCVD. ${ }^{3-6}$ In the Internal Medicine Clinic, we identified a gap of evidence-based treatment for hyperlipidaemia; less than $1 \%$ of active, eligible patients had ASCVD risk scores documented in electronic health record using the ASCVD risk calculator. These data indicate that evidence-based treatment for hyperlipidaemia using ACC/AHA guidelines were underused in the Internal Medicine Clinic.

\section{Background}

The ACC/AHA guidelines focus on ASCVD risk reduction, advocating for the use of evidence-based doses of HMG-CoA-enzyme reductase inhibitors (statins) as first-line therapy and using a Pooled Cohort Equation risk calculator to calculate a patient's 10-year risk score, which is used to guide initiation of statin therapy. ${ }^{3-8}$ Based on this 10-year percent risk, patients are stratified into highrisk, moderate-risk or low-risk categories and are accordingly started on high-intensity or medium-intensity statin medications. ${ }^{2-6}$ Therefore, identification of patients who are eligible to receive statin therapy as per current ACC/AHA guidelines is not possible without calculation of the Pooled Cohort ASCVD risk score.

Primary prevention of ASCVD includes a heart healthy lifestyle ${ }^{9-12}$ as well as the use of medications, including lipid lowering statins, which target modifiable cardiovascular risk factors and have been shown to reduce coronary artery disease, stroke and other cardiovascular diseases. ${ }^{9} 10$ There are multiple comorbidities that are significant risk factors for coronary artery disease, including obesity and hyperlipidaemia, and predispose patients to future cardiovascular events. Over $70 \%$ of the Internal Medicine Clinic patient population has a diagnosis of hypertension and obesity (body mass index of 30 or greater). The majority of these patients have associated comorbidities that include type 2 diabetes mellitus and nicotine dependence. Many of these patients have undiagnosed 
hyperlipidaemia and are at risk for major cardiovascular mortality and morbidities such as coronary artery disease, stroke, heart failure and premature death. ${ }^{13-17}$ Therefore, diagnosing comorbidities and implementing statin therapy based on an ASCVD risk score is crucial to reduce cardiovascular morbidity and mortality. ${ }^{3-6}$ Since the majority of the Internal Medicine Clinic patients did not have ASCVD risk score assessment, the aim of this quality improvement project was to increase ASCVD risk score assessment in patients between the ages of 40 and 75 years from a baseline rate of less than $1 \%$ to $10 \%$, within 12 months. The ultimate goal was improving treatment of blood cholesterol for the primary prevention of ASCVD in the Internal Medicine Clinic.

\section{METHODS}

Setting

This quality improvement project was conducted in an academic Internal Medicine Clinic, located within a tertiary care safety net hospital. The multidisciplinary care team included 5 attending physicians, 40 rotating residents (Internal Medicine Residency Program of the University at Buffalo, State University of New York), nurses (Registered Nurses and licensed practical nurse), a social worker and administrative staff. The population is mostly urban and underserved patients. The Internal Medicine Clinic provides longitudinal primary care. The monthly average patients' visits were about 700 , with a consistent show rate of $80 \%$.

We create an electronic patient database, in collaboration with the information technology department, using electronic health records. We included male and female patients, between the ages of 18 and 75 years, seen from June 2015 to June 2016 in the Internal Medicine Clinic. The baseline ASCVD risk scores were less than $1 \%$ in eligible patients, calculated by retrospective review of the health record database for patients seen within 6 months. Prior to initiation of this project, Internal Medicine Clinic patients were diagnosed and treated for hyperlipidaemia based on Adult Treatment Panel (ATP) III guidelines. ${ }^{18-20}$ Patients were eligible for a lipid profile blood test if they did not have a lipid profile completed within the past 5 years. Patients were eligible for ASCVD risk score calculation if they had no documented ASCVD risk score in their electronic health record at any previous visits. During 12 months of this quality improvement, patients were counted more than once if they were eligible during any clinic visit.

\section{Measurements}

The outcome measures were percentage of patients with documented ASCVD risk score in electronic health records, and percentage of new prescriptions for statins based on ACC/AHA guidelines. Process measures included: lipid profile order rate in eligible patients and lipid profile completion rate. An increase in patient wait time, blood test and medications costs were the balanced measures. We set a low improvement target for the outcome measure due to the following reasons: (1) Patients in the Internal Medicine Clinic had multiple comorbidities, therefore there was limited follow-up time (20-30 $\mathrm{min}$ ) and we anticipated a lack of sufficient time to address lipid ASCVD risk documentation in every patient. (2) We anticipated multiple barriers to the acceptance of fasting lipid profile blood test and treatment of hyperlipidaemia as the majority of Internal Medicine Clinic patients were underserved, uninsured and a vulnerable population.

\section{Data analysis}

We analysed data from the electronic patient database and created weekly statistical process control (SPC) charts for the process measure and outcome measure.

\section{Design}

We designed our Aim statement using SMART (Specific, Measurable, Achievable, Results, Timely) goal. ${ }^{21} \mathrm{We}$ also used the STEEEP (Safe, Timely, Effective, Efficient, Equitable, Patient-centered) model from the Institute of Medicine $^{22}$ to design this project as well as the PlanDo-Study-Act (PDSA) model for quality improvement . ${ }^{23}$ A root cause analysis in a small group discussion setting with a multidisciplinary team was performed to identify barriers to the acceptance of lipid profile blood tests, calculating ASCVD risk scores and treatment. Materials/ methods, provider and patient-related barriers were identified (figure 1). We addressed the identified barriers and developed interventions to improve the ASCVD risk documentation and treatment based on ACC/AHA blood lipid guidelines (figure 2). Initiation of statins for the primary prevention of ASCVD involved a shared decision making process between the provider and the patient during which the benefits of statin therapy, adverse effects, potential drug-drug interactions as well as patient preferences were discussed.

\section{Strategy}

This quality improvement was conducted for a period of 12 months (from June 2015 to June 2016). Forty residents were divided into five cohorts; each cohort had eight residents. Internal medicine residents rotated in the ambulatory Internal Medicine Clinic every 5 weeks, residents spent 1 week in the continuity of care clinic in the Internal Medicine Clinic. Forty residents, five attending physicians and ancillary Internal Medicine Clinic staff participated in this quality improvement project. We plotted weekly results from the database every 5 weeks during every new cohort of residents.

\section{PDSA cycle 1}

(June-July 2015) Integration of customised workflow in electronic health records: In collaboration with the information technology department, we created a customised electronic health record template that integrated ASCVD risk calculation. There was no automated ASCVD risk calculator in the electronic health record. A new 


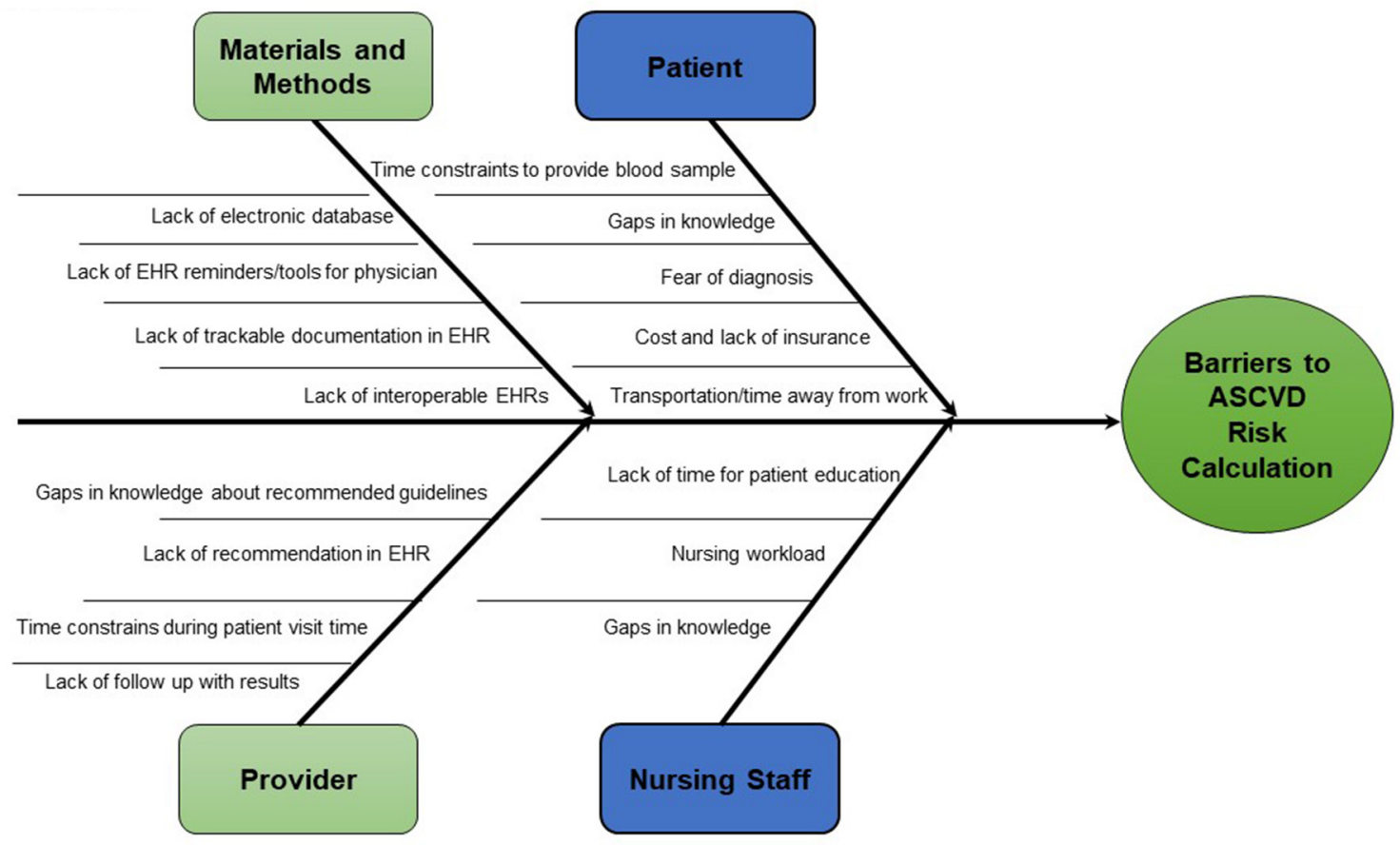

Figure 1 Fishbone diaphragm: root cause analysis identifying barriers to hyperlipidaemia screening and ASCVD risk calculation. ASCVD, atherosclerotic cardiovascular disease; EHR, electronic health record; IMC, internal medicine clinic; MOA, medical office assistant; QI, quality improvement.

customised workflow included calculation of ASCVD risk using an ASCVD risk calculator in a separate application outside of the electronic health record and manual entry of calculated ASCVD risk score in the electronic health record as a structured data entry. This step was crucial to generate trackable data from electronic health records. ASCVD documentation of structured data was incorporated into the 'finding' section of the electronic health record. We also created a customised check list in the electronic health record to document patient-related

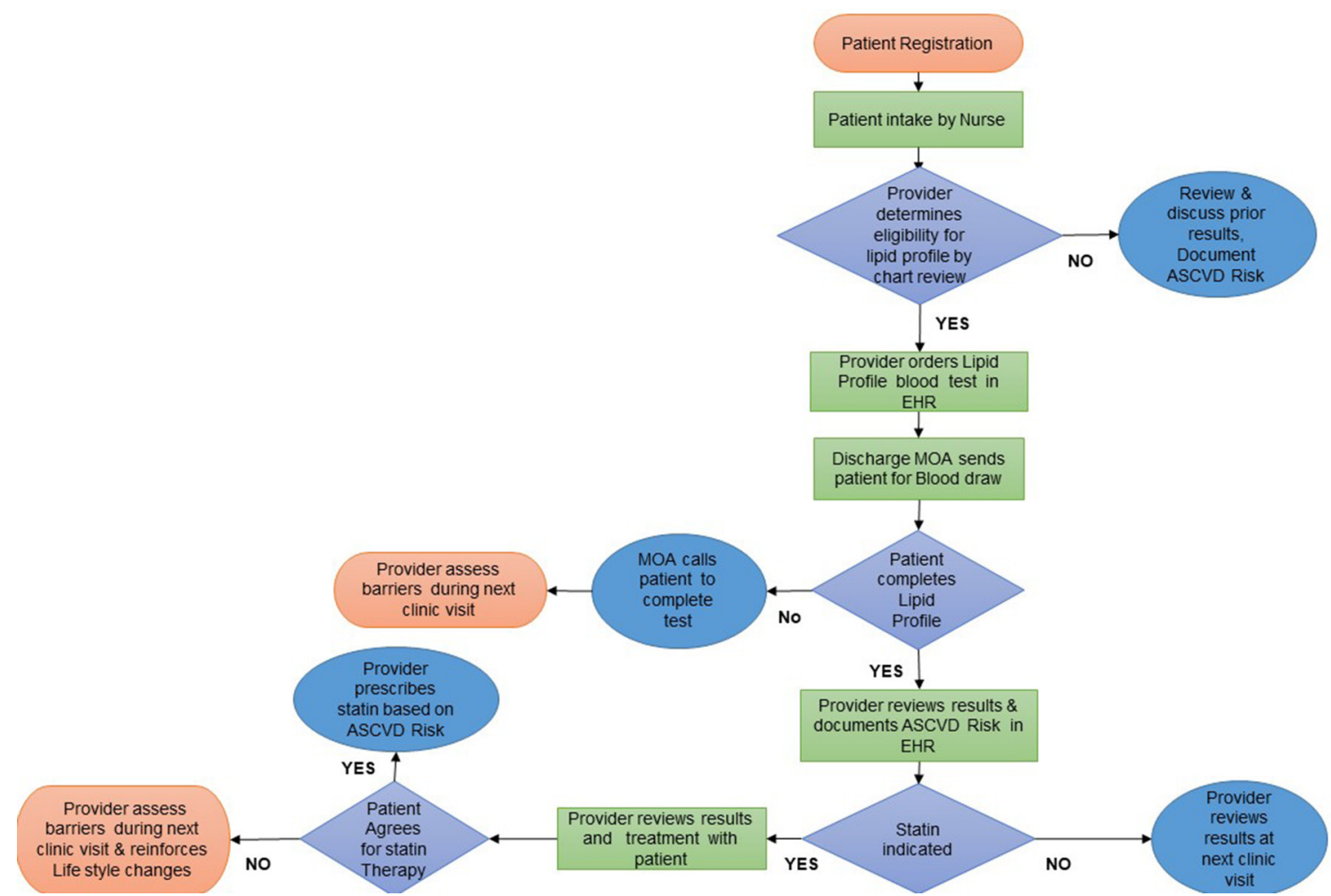

Figure 2 Process flow map for hyperlipidaemia screening and ASCVD risk calculation. ASCVD, atherosclerotic cardiovascular disease. EHR, electronic health record; MOA, medical office assistant. 
barriers to acceptance of statin therapy. We included barriers of communication, knowledge, transportation, understanding of diagnosis and cost of medication. This innovative electronic health record workflow generated structured documentation under 'Health Management'. Physicians documented barriers to acceptance of a recommended statin therapy based on a calculated ASCVD risk score. Patients were referred to a social worker when physician identified 'cost' of the medication as the barrier to acceptance of statin therapy.

\section{PDSA cycle 2}

(August-December 2015) Resident Education about lipid guidelines and ASCVD workflow in electronic health records: We identified a gap in residents' knowledge regarding 2013 ACC/AHA lipid guidelines. The quality improvement resident leader and attending physician champion provided training and education to the Internal Medicine Clinic physicians and reviewed a new customised electronic health record workflow for ASCVD risk documentation. The team discussed guidelines and workflow in a small group discussion format using a PowerPoint presentation.

\section{PDSA cycle 3}

(January-March 2016) Refresher Training for Residents and Chart Alert: We provided refresher training to internal medicine residents. The quality improvement team assessed various physician-related barriers to documentation of ASCVD risk scores in the electronic health record. Physicians were instructed to create manual chart alerts to remind physicians to perform ASCVD risk calculation.

\section{PDSA cycle 4}

(April-June 2016) Patient Education and Physician Reminder: In order to improve physician reminders as well as patient awareness, heart-shaped reminder pamphlets were placed in the physicians' task boxes and posted in patient's examination rooms (figure 3).

\section{RESULTS}

Process measures

Lipid profile order rates

We were unable to report accurate results of lipid order rates due to a deficiency in the electronic database. Therefore, we have reported imperfect estimates. A $50 \%$ lipid profile order rate was calculated in eligible patients.

\section{Lipid profile completion rate}

The mean (average) lipid profile completion rate in eligible patients was $18 \% \quad(\mathrm{n}=313 / 1691$ patient visits $)$ (figure 4A). The completion rate is based on the total number of patients' visits that were eligible for a lipid profile order. We estimated only a $50 \%$ completion rate of fasting lipid profiles once physicians ordered the blood test. This is based on anecdotal reports of physicians. In our database, it was not possible to accurately identify orders of lipid profiles. The significant variation seen in relation to PDSA cycles was not sustainable.

\section{Outcome measures}

\section{ASCVD risk completion rate}

The average ASCVD risk calculation completion rate was $14.2 \%$ ( $n=364 / 2565$ patients). The mean ASCVD risk completion rate was $4.0 \%$ ( $\mathrm{n}=364 / 9105$ patient visits) within a 12-month period, plotted in a weekly SPC chart (figure 4B). During the first PDSA cycle, the ASCVD risk calculation completion rate was very low except for a $12 \%$ increase during the week of 5 July to 11 July 2015. This specific weekly increase may be explained by active participation of team residents that were assigned to implement this quality improvement project as residents rotated every 5 weeks and spent 1 week in the clinic during the ambulatory continuity care clinic rotation. However, this

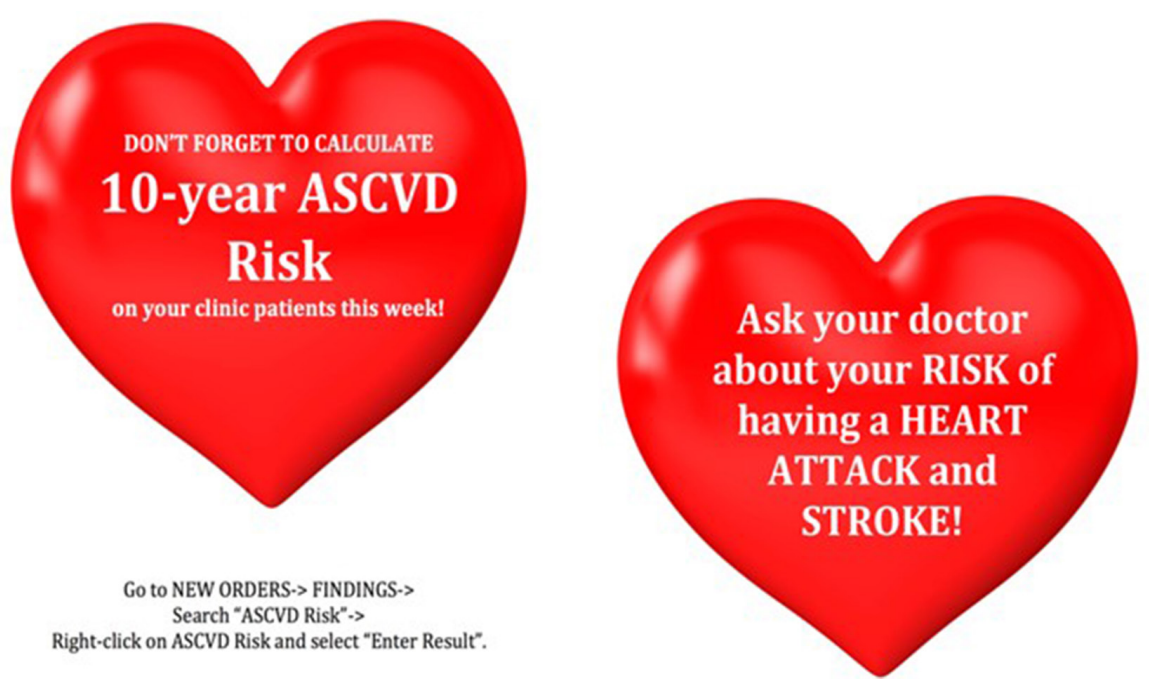

Figure 3 Handouts reminding physicians about calculating a 10-year ASCVD risk score and patients asking about their risk of heart attack and stroke. ASCVD, atherosclerotic cardiovascular disease. 

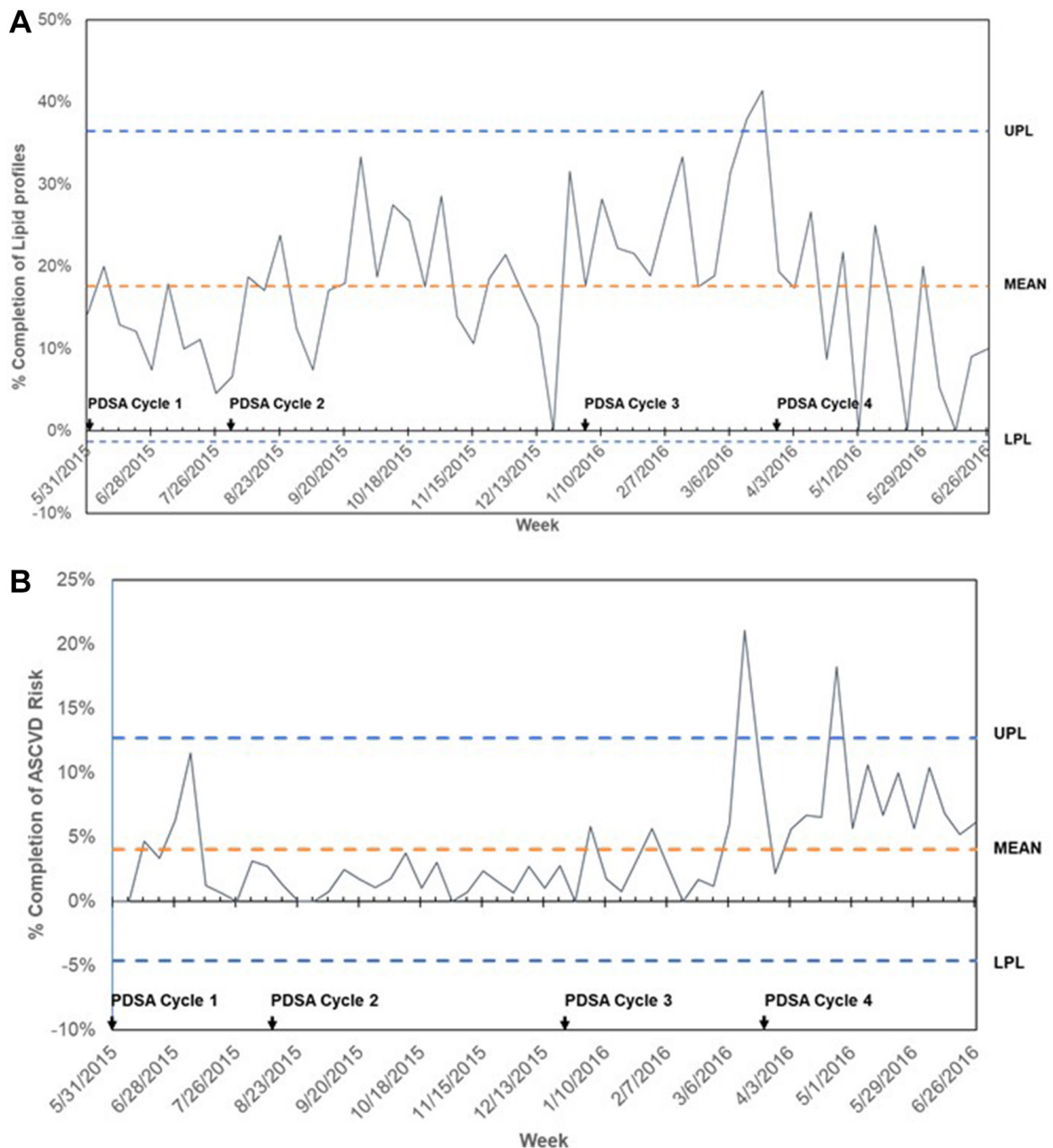

Figure 4 (A) Weekly SPC chart: ASCVD risk calculation completion rate. (B) Weekly SPC chart: lipid completion rate. ASCVD, atherosclerotic cardiovascular disease; LCL, lower control limit; PDSA, Plan-Do-Study-Act; SPC, statistical process control; UCL, upper control limit.

increase was not sustainable. The ASCVD risk calculation completion rate was very low; this may be explained by the lack of physician's knowledge about ACC/AHA lipid guidelines and unawareness of the new electronic health record workflow for ASCVD risk completion, we based these conclusions on residents' anecdotal reports. During the second PDSA cycle, we did not achieve any significant increase in ASCVD risk calculation completion rates. During the third PDSA cycle, the ASCVD risk calculation completion rate was highly variable, with a $21 \%$ increase during the week of 13 March to 19 March 2016. During the 4th PDSA cycle, we achieved unsustainable variability with an $18 \%$ increase during the week of 24 April to 30 April 2016. We had difficulty in sustaining the improvement during the entire project; however, we were able to sustain an ASCVD risk score completion rate of $33 \%$ ( $\mathrm{n}=644 / 1949$ patients) during the postproject 1-year period. Several explanations for the sustainability of this ASCVD rate increase after the project improvement period include: (1) Continuous quality improvement of ASCVD risk assessment was integrated as a standard of care in the physician work flow as a part of routine screening tests in the -Internal Medicine Clinic.
(2) Annual and ongoing education of new interns and residents regarding ACC/AHA lipid lowering guidelines was done. (3) Reminder and reinforcement was given to residents by an actively engaged supervising Attending Physician Champion to address ASCVD risk score during case presentations at the time of clinic visit.

\section{New prescriptions for statins}

A total of 359 new statin prescriptions based on ACC/ AHA guidelines were initiated in the Internal Medicine Clinic population for primary prevention of ASCVD due to this quality improvement project.

\section{Balance measures}

Increase in patient wait time

During this quality improvement, we did not observe a significant increase in patient wait time during clinic visits. The extra time required for the physician to perform ASCVD risk calculation and to explain the calculated risk to the patient did not result in any patient backlog during clinic visits. This was determined by anecdotal reports of lack of patient complaints for a long wait time and lack of overtime for clinic staff during this project. The baseline 
time for the cycle time of a follow-up clinic visit was an average of 1 hour from start of the registration to patient discharge. We did not measure actual cycle time during the project.

\section{Cost of blood test and medications}

Internal Medicine Clinic physicians identified blood test and medication costs as a minor barrier. The cost of statin medication was documented by physicians in the electronic health record under barriers for acceptance of lipid therapy; however, we were unable to retrieve an accurate percentage of patients who refused lipid therapy due to cost reasons. During follow-up clinic visits, patients reported to the physicians that requirement of fasting blood sample for lipid profile was the barrier for the completion of lipid profile.

\section{Summary}

In the Internal Medicine Clinic, we identified a gap in the implementation of AHA/ACC blood cholesterol guidelines, with a major deficiency in ASCVD risk scores assessment, therefore, we implemented a quality improvement project to increase the percentage of patients with calculated ASCVD risk scores. The ultimate goal was to improve treatment of blood cholesterol for primary prevention of ASCVD. The mean ASCVD risk completion rate was $14.2 \%$ ( $\mathrm{n}=364 / 2565$ patients) within 12 months. ASCVD risk score assessment completion rate was sustainable; achieving 33\% ( $\mathrm{n}=644 / 1949$ patients) 1 year post project completion. Meaningful impact was demonstrated in the rate of ASCVD risk calculation from the various PDSA cycles. Manual entry of ASCVD risk calculation in electronic health record by physicians in the electronic health record resulted in a sustainable increase in the diagnosis and treatment of hyperlipidaemia in the Internal Medicine Clinic. The average lipid profile completion rate was $18 \%$ in eligible patients. The significant variation seen in relation to various PDSA cycles was not sustainable for the process measure of lipid completion rate. Low lipid completion rates and various physician related barriers to acceptance of the ASCVD risk calculation resulted in a slow increase in ASCVD risk score assessment rate. This quality improvement did not result in an increase in opportunity costs.

This quality improvement project has been translated to an increase in the number statin prescriptions for primary prevention of ASCVD in the Internal Medicine Clinic. We learned several important lessons. Multidisciplinary team engagement and root cause analysis to identify various barriers were the key components responsible for the success of this quality improvement project. Lack of automated electronic health record algorithm support to determine eligibility for lipid testing, calculating and documenting ASCVD risk, and clinical decision support to determine the treatment plan were found to be the greatest system barriers. Our electronic health record did not have an automated and integrated ASCVD risk calculator. The addition of this feature was considered an enhancement to the existing electronic health record, with an additional cost. Automatically calculated risk scores can be used successfully in population health management. ${ }^{24}$ Despite these barriers, an innovative workflow was introduced within the electronic health record which resulted in surpassing our aim of a $10 \%$ increase in ASCVD risk score assessment within 12 months. Physician's barriers to the acceptance of ASCVD risk assessment included: lack of electronic chart alerts and lack of extra time needed to complete the ASCVD risk score assessment during the limited clinic visit while addressing multiple chronic diseases. The continuous feedback from quality improvement team members was crucial to identify appropriate interventions. This quality improvement project has resulted in an increase in the use of ASCVD risk calculator and initiating a patient-centred approach for the management of blood cholesterol with an ultimate goal of primary prevention of ASCVD in the clinic population.

\section{Interpretation}

In an effort to reduce the global burden of ASCVD, the ACC/AHA released guidelines in 2013 for treatment of blood cholesterol for reducing the risk of ASCVD in adults. ${ }^{3-6}$ These guidelines replaced the 2004 National Cholesterol Education Program (NCEP) ATP III guidelines that focused on low-density lipoprotein cholesterol (LDL-C) levels for prevention and treatment of coronary artery disease. ${ }^{20}$ Instead, the current guidelines have shifted the emphasis on management of risk of ASCVD in adults. ${ }^{3-6}$ Estimated risk is based on the pooled cohort equation to predict the risk of having a cardiovascular event. Patients are stratified into high-risk, moderate-risk or low-risk categories and accordingly started on high-intensity or medium-intensity statin medications. Additionally, for high-risk patients with clinical ASCVD, LDL cholesterol $\geq 190 \mathrm{mg} / \mathrm{dL}$, diabetes history and estimated 10 -year ASCVD risk $\geq 7.5 \%$ should be on a high-intensity statin..$^{3-6}$ Prior to this quality improvement, Internal Medicine Clinic patients were treated for hyperlipidaemia based on ATP III guidelines. Current evidence validates the efficacy of implementation of therapies based on ASCVD risk scores. ${ }^{785-29}$ However, less than $1 \%$ of the Internal Medicine Clinic population had ASCVD risk scores completed; therefore, implementation of the current evidence-based treatment guidelines is the most effective way to increase primary prevention and management of ASCVD in the Internal Medicine Clinic.

\section{Limitations}

This quality improvement project has several limitations: (1) We were unable to retrieve the rate of new statin prescriptions and rate of modification (change in dose or intensity) of prescriptions for primary prevention of ASCVD based on ACC/AHA guidelines in Internal Medicine Clinic. (2) This quality improvement was conducted in a safety-net primary care clinic in patients with multiple comorbidities. These results are not generalisable to 
other settings. (3) We did not analyse the results of specific types of patient-related barriers to the acceptance of statin therapy.

\section{CONCLUSION}

A team-based approach including patient's engagement was critical in the success of this quality improvement project. We have implemented ASCVD risk score assessment as a standard of care in the Internal Medicine Clinic. We plan to increase ASCVD risk score assessment substantially and make interventions sustainable by shifting the responsibility of ASCVD risk calculation from the physician to the nursing staff, and creation of a chart alert to remind physicians during previsit planning. These steps may increase physician's efficiency and physician's acceptance of ASCVD risk score discussion with patients, and offering of evidence-based treatment for primary prevention of ASCVD in clinic patients. The next phase of this study will focus on ensuring appropriate of statin prescriptions (dose and recommended intensity) based on ACA/AHA guidelines. With regard to future directions to improve and continue our efforts towards primary prevention of ASCVD, we are planning to monitor therapeutic responses as well as adherence to statin therapy by repeating lipid panels to confirm the percent decrease in serum low-density lipoprotein within 6 months to 1 year after initiation of statin therapy.

Acknowledgements We would like to thank the Administrative Staff, IT Department and Clinic Nursing Staff of ECMC.

Contributors SYB: Study oversight, study concept and design, acquisition of data, analysis and interpretation of data, drafting of manuscript, critical revision of the manuscript for important intellectual content and finalisation of manuscript. $\mathrm{AB}$ : Acquisition of data, analysis and interpretation of data. PS: Acquisition of data, analysis and interpretation of data. JLR: Interpretation of data, drafting of manuscript, critical revision of the manuscript for important intellectual content and finalisation of manuscript.

Funding The authors have not declared a specific grant for this research from any funding agency in the public, commercial or not-for-profit sectors.

Competing interests None declared.

Patient consent Not required.

Ethics approval Based on local policy, this work was deemed as an improvement study and not a research study on human subjects as patients were not affected directly and their treatment was not altered nor were they given an experimental or novel therapy. The local research policy was met and ethical approval was not required.

Provenance and peer review Not commissioned; externally peer reviewed.

Data sharing statement An electronic patient database is available, contact authors for access.

Open access This is an open access article distributed in accordance with the Creative Commons Attribution Non Commercial (CC BY-NC 4.0) license, which permits others to distribute, remix, adapt, build upon this work non-commercially, and license their derivative works on different terms, provided the original work is properly cited, appropriate credit is given, any changes made indicated, and the use is non-commercial. See: http://creativecommons.org/licenses/by-nc/4.0/.

\section{REFERENCES}

1. WHO. Cardiovascular diseases (CVDs). 2017. http://www.who.int/ mediacentre/factsheets/fs317/en/
2. Ray KK, Kastelein JJ, Boekholdt SM, et al. The ACC/AHA 2013 guideline on the treatment of blood cholesterol to reduce atherosclerotic cardiovascular disease risk in adults: the good the bad and the uncertain: a comparison with ESC/EAS guidelines for the management of dyslipidaemias 2011. Eur Heart J 2014;35:960-8.

3. Stone NJ, Robinson JG, Lichtenstein A. The new cholesterol treatment guidelines.. N Engl J Med 2014;370.

4. Stone NJ, Robinson JG, Lichtenstein AH, et al. 2013 ACC/ AHA guideline on the treatment of blood cholesterol to reduce atherosclerotic cardiovascular risk in adults: a report of the American College of Cardiology/American Heart Association Task Force on Practice Guidelines. J Am Coll Cardiol 2014;63:2889-934.

5. Stone NJ, Robinson JG, Lichtenstein AH, et al. 2013 ACC/ AHA guideline on the treatment of blood cholesterol to reduce atherosclerotic cardiovascular risk in adults: a report of the American College of Cardiology/American Heart Association Task Force on Practice Guidelines. Circulation 2014;129(25 Suppl 2):S1-45.

6. Stone NJ, Robinson JG, Lichtenstein AH, et al. Treatment of blood cholesterol to reduce atherosclerotic cardiovascular disease risk in adults: synopsis of the 2013 American College of Cardiology/ American Heart Association cholesterol guideline. Ann Intern Med 2014;160:339-343-43.

7. Muntner P, Colantonio LD, Cushman M, et al. Validation of the atherosclerotic cardiovascular disease pooled cohort risk equations. JAMA 2014:311:1406-15.

8. Nooe A, Edwards MK, Addoh O, et al. Convergent validity of the ACC/AHA pooled cohort equations in associating with health-related quality of life among adults in the United States. Health Promot Perspect 2017;7:42-6.

9. Eckel RH, Jakicic JM, Ard JD, et al. 2013 AHA/ACC guideline on lifestyle management to reduce cardiovascular risk: a report of the American College of Cardiology/American Heart Association Task Force on Practice Guidelines. J Am Coll Cardiol 2014:63:2960-84.

10. Eckel RH, Jakicic JM, Ard JD, et al. 2013 AHA/ACC guideline on lifestyle management to reduce cardiovascular risk: a report of the American College of Cardiology/American Heart Association task force on practice guidelines. Circulation 2014;129(25 Suppl 2):S76-99.

11. Anderson TJ. Importance of optimization of cardiovascular risk factors and lifestyle behaviours. Can J Cardiol 2017;33:1221-2.

12. Doughty KN, Del Pilar NX, Audette A, et al. Lifestyle medicine and the management of cardiovascular disease. Curr Cardiol Rep 2017; $19: 116$

13. Burkhardt R. Hyperlipidemia and cardiovascular disease: reinforcement for 'lower is better'. Curr Opin Lipidol 2015;26:468-9.

14. Last AR, Ference JD, Menzel ER. Hyperlipidemia: drugs for cardiovascular risk reduction in adults. Am Fam Physician 2017;95:78-87.

15. Malloy MJ, Kane JP. Hyperlipidemia and cardiovascular disease. Curr Opin Lipidol 2012;23:591-2.

16. Nelson RH. Hyperlipidemia as a risk factor for cardiovascular disease. Prim Care 2013;40:195-211.

17. Tietge UJ. Hyperlipidemia and cardiovascular disease: inflammation, dyslipidemia, and atherosclerosis. Curr Opin Lipidol 2014;25:94-5.

18. Rodríguez-Ortiz D, Reyes-Pérez A, León P, et al. Assessment of two different diagnostic guidelines criteria (National Cholesterol Education Adult Treatment Panel III [ATP III] and International Diabetes Federation [IDF]) for the evaluation of metabolic syndrome remission in a longitudinal cohort of patients undergoing Roux-en-Y gastric bypass. Surgery 2016;159:1121-8.

19. Talwalkar PG, Sreenivas CG, Gulati A, et al. Journey in guidelines for lipid management: From adult treatment panel (ATP)-I to ATP-III and what to expect in ATP-IV. Indian J Endocrinol Metab 2013;17:628-35.

20. National Cholesterol Education Program (NCEP) Expert Panel on Detection, Evaluation, and Treatment of High Blood Cholesterol in Adults (Adult Treatment Panel III). Third Report of the National Cholesterol Education Program (NCEP) Expert Panel on Detection, Evaluation, and Treatment of High Blood Cholesterol in Adults (Adult Treatment Panel III) final report. Circulation 2002;106:3143-421.

21. UHR, Employee Development. Writing S.M.A.R.T. Goals. 2009. http:// www.hr.virginia.edu/uploads/documents/media/Writing_SMART_ Goals.pdf

22. Transforming healthcare delivery through patient-centered care. 2011. http://www.amga.org/wcm/Pl/Acclaim/2011/healthTexas.pdf

23. Taylor MJ, McNicholas C, Nicolay C, et al. Systematic review of the application of the plan-do-study-act method to improve quality in healthcare. BMJ Qual Saf 2014;23:290-8.

24. North F, Fox S, Chaudhry R. Clinician time used for decision making: a best case workflow study using cardiovascular risk assessments and Ask Mayo Expert algorithmic care process models. BMC Med Inform Decis Mak 2016;16:96. 
25. Chia YC, Lim HM, Ching SM. Validation of the pooled cohort risk score in an Asian population - a retrospective cohort study. BMC Cardiovasc Disord 2014;14:163.

26. Qureshi WT, Michos ED, Flueckiger P, et al. Impact of replacing the pooled cohort equation with other cardiovascular disease risk scores on atherosclerotic cardiovascular disease risk assessment (from the Multi-Ethnic Study of Atherosclerosis [MESA]). Am J Cardiol 2016;118:691-6.

27. Schiros CG, Denney TS, Gupta H. Interaction analysis of the new pooled cohort equations for 10-year atherosclerotic cardiovascular disease risk estimation: a simulation analysis. BMJ Open 2015;5:e006468.

28. Topel ML, Shen J, Morris AA, et al. Comparisons of the Framingham and pooled cohort equation risk scores for detecting subclinical vascular disease in blacks versus whites. Am J Cardiol 2018;121:564-9.

29. Tralhão A, Ferreira AM, Gonçalves PA, et al. Accuracy of pooledcohort equation and SCORE cardiovascular risk calculators to identify individuals with high coronary atherosclerotic burden implications for statin treatment. Coron Artery Dis 2016;27:573-9. 